\title{
ERRATUM
}

\section{Hawking Radiation of Dirac Particles in a Variable-Mass Kerr Space-Time [Gen. Rel. Grav. 33 (2001) 1181-1195]}

\section{S. Q. Wu ${ }^{1}$ and X. Cai ${ }^{1}$}

Received April 10, 2002

In the appendix, the second one and the fourth one of Eq. (A2) should be

$$
\begin{aligned}
d \boldsymbol{n}= & \left(\frac{r \Delta}{\Sigma^{2}}-\frac{r-M}{\Sigma}\right) \boldsymbol{n} \wedge \boldsymbol{l}-\frac{i \dot{M} r a \sin \theta}{\sqrt{2} \Sigma}\left(\frac{\boldsymbol{m}}{\rho}-\frac{\overline{\boldsymbol{m}}}{\rho^{*}}\right) \wedge \boldsymbol{l} \\
& -\frac{i a \cos \theta \Delta}{\Sigma^{2}} \boldsymbol{m} \wedge \overline{\boldsymbol{m}}, \\
d \overline{\boldsymbol{m}}= & -\frac{\sqrt{2} i r a \sin \theta}{\Sigma \rho} \boldsymbol{n} \wedge \boldsymbol{l}-\frac{1}{\rho^{*}}\left(\boldsymbol{n}-\frac{\Delta}{2 \Sigma} \boldsymbol{l}\right) \wedge \overline{\boldsymbol{m}}-\frac{1}{\sqrt{2} \rho} \\
& \times\left(\cot \theta-\frac{i a \sin \theta}{\rho}\right) \boldsymbol{m} \wedge \overline{\boldsymbol{m}} .
\end{aligned}
$$

We apologize for this typesetting error.

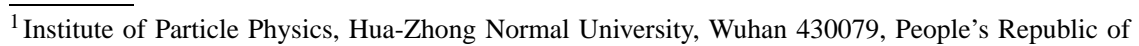
China; e-mail: sqwu@iopp.ccnu.edu.cn; xcai@ccnu.edu.cn
} 